This item was submitted to Loughborough's Research Repository by the author.

Items in Figshare are protected by copyright, with all rights reserved, unless otherwise indicated.

\title{
Efficient three dimensional modelling of additive manufactured textiles
}

PLEASE CITE THE PUBLISHED VERSION

http://www.emeraldinsight.com/journals.htm?issn=1355-2546

\section{PUBLISHER}

(c) Emerald Group Publishing Limited

\section{VERSION}

SMUR (Submitted Manuscript Under Review)

\section{LICENCE}

CC BY-NC-ND 4.0

\section{REPOSITORY RECORD}

Bingham, Guy A., and Richard J.M. Hague. 2019. "Efficient Three Dimensional Modelling of Additive Manufactured Textiles". figshare. https://hdl.handle.net/2134/12257. 
This item was submitted to Loughborough's Institutional Repository (https://dspace.lboro.ac.uk/) by the author and is made available under the following Creative Commons Licence conditions.

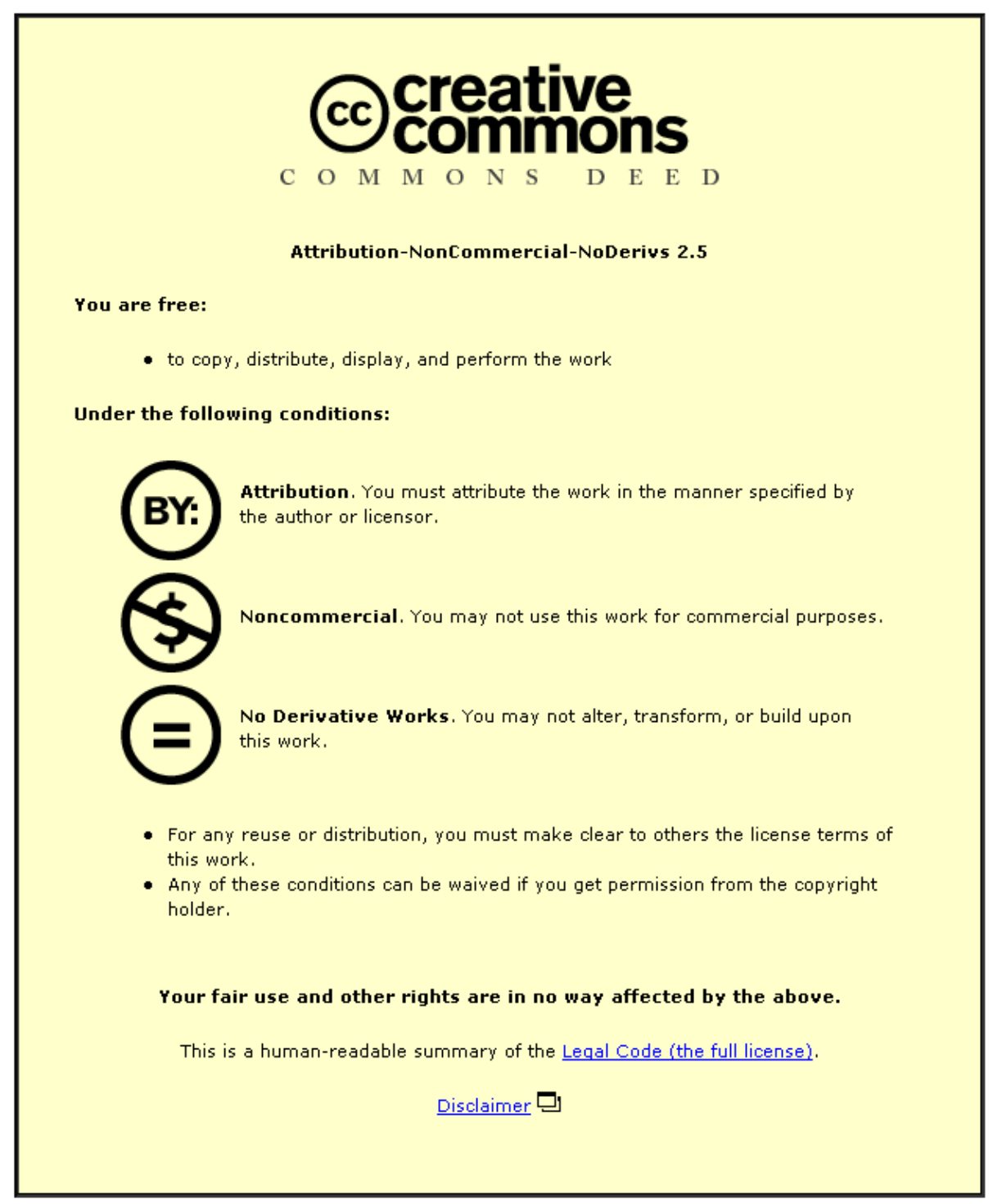

For the full text of this licence, please go to: http://creativecommons.org/licenses/by-nc-nd/2.5/ 


\section{Efficient Three Dimensional Modelling of Additive Manufactured Textiles}

Dr. Guy A. Bingham (corresponding author)

Loughborough Design School

Loughborough University

Leicestershire, UK

LE11 3TU

g.a.bingham@lboro.ac.uk

Dr Guy Bingham is a Lecturer in the Design School at Loughborough University and a member of the Design Practice Research Group. His research focus is concerned with the design opportunities and implications of Additive Manufacturing.

Prof Richard Hague

Wolfson School of Mechanical and manufacturing Engineering

Loughborough University

Leicestershire, UK

LE11 3TU

r.hague@lboro.ac.uk

Short Bio 


\title{
Efficient Three Dimensional Modelling of Additive Manufactured Textiles
}

\begin{abstract}
Purpose -

To investigate, develop and validate a three dimensional modelling strategy for the efficient generation of conformal textile data suitable for Additive Manufacture.

\section{Design/methodology/approach -}

A series of Additive Manufactured textiles samples were modelled using currently available Computer Aided Design software to understand the limitations associated with the generation of conformal data. Results of the initial three dimensional modelling processes informed the exploration and development of a new dedicated efficient modelling strategy that was tested to understand its capabilities.
\end{abstract}

\section{Findings -}

The research demonstrates the dramatically improved capabilities of the developed three dimensional modelling strategy, over existing approaches by accurately mapping complex geometries described as STL data to a mapping mesh without distortion and correctly matching the orientation and surface normal.

\section{Originality/value -}

To date the generation of data for AM textiles has been seen as a manual and timeconsuming process. The research presents a new dedicated methodology for the efficient generation of complex and conformal Additive Manufactured textile data that will underpin further research in this area.

Keywords: Additive Manufacturing, Textile, 3D Modelling, CAD

Paper type: Research paper 


\subsection{Introduction}

Textiles structures realised by Additive Manufacturing (AM) techniques, known as AM textiles [Bingham 2007] (demonstrated in Figure 1), have received increasing attention during the previous decade. Due to their potential to significantly improve on both the geometric complexity and functionality available from conventional fibre-based textiles, AM textiles present an opportunity to design and develop novel solutions for high-performance and smart textile applications. AM textiles also provide the ability to produce net-shape textile artefacts that can allow the development of personalised smart and high-performance textile applications from a variety of materials currently being processed by AM technologies.

Research in this area has mainly concentrated on the development of efficient modelling strategies for the creation of the three dimensional (3D) Computer Aided Design (CAD) data required for their manufacture by AM techniques [Bingham et al 2007, Bingham 2007]. However, additional research has also been undertaken to address the mechanical properties, design and possible applications of these novel complex structures [Crookston et al 2008, Johnson et al 2011].

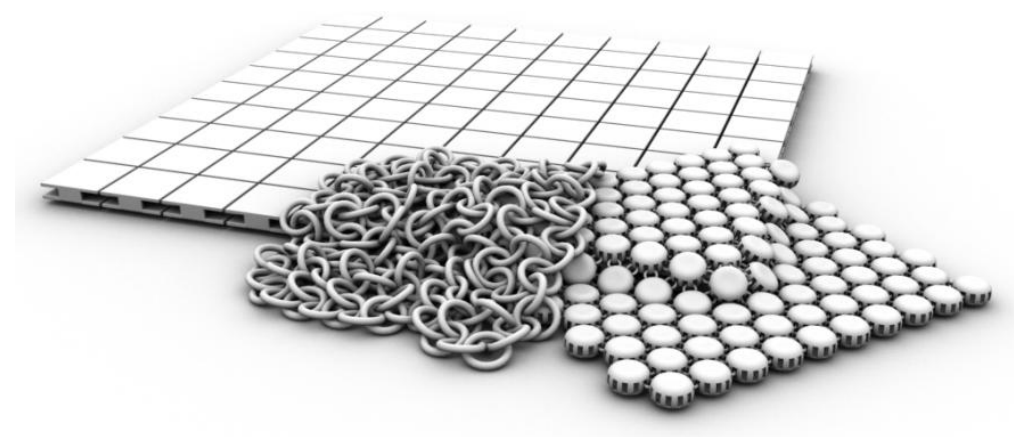

Figure 1: Examples of AM textiles

While the motivation exists for the wider-scale adoption of these novel complex structures, practical access to an efficient 3D modelling strategy required for their manufacture limits their applications. The research presented here discusses the issues surrounding the 3D modelling of complex AM textiles and proposes a dedicated methodology for the efficient generation of complex and conformal AM textile artefacts. 


\subsection{Initial AM textile modelling with commercial systems}

The modelling of individual AM textile link structures using currently available 3D CAD systems is relatively simple for expert users and complex geometric designs can be generated with relative ease. The use of 3D CAD systems can be expanded further for the generation of planar samples of AM textile using a simple planar 'Array' or 'Patterning' function common to most current 3D CAD systems. Planar samples can be generated as either quadrilateral-based or triangular-based patterned arrays as demonstrates in Figure 2.
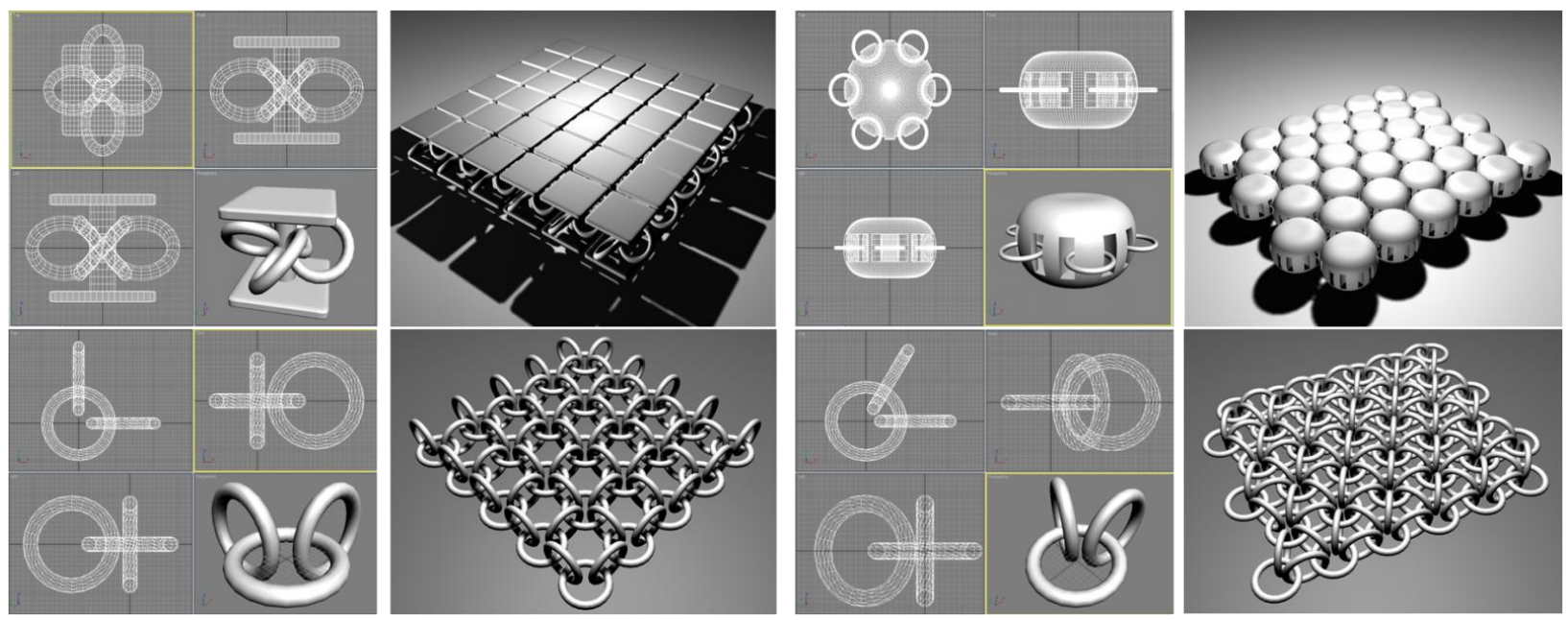

Figure 2: AM textile samples, Left - quadrilateral-based, right - triangular-based

The limitations associated with current 3D CAD systems relate to the capability of generating conformal or net-shape AM textile artefacts. While it is possible to generate simple net-shape AM textiles (e.g. cylindrical), the generation of more complex net-shape AM textiles, such as hemispherical samples, is problematic and results in incorrect tessellation of the individual link structures, also demonstrated in Figure 3.
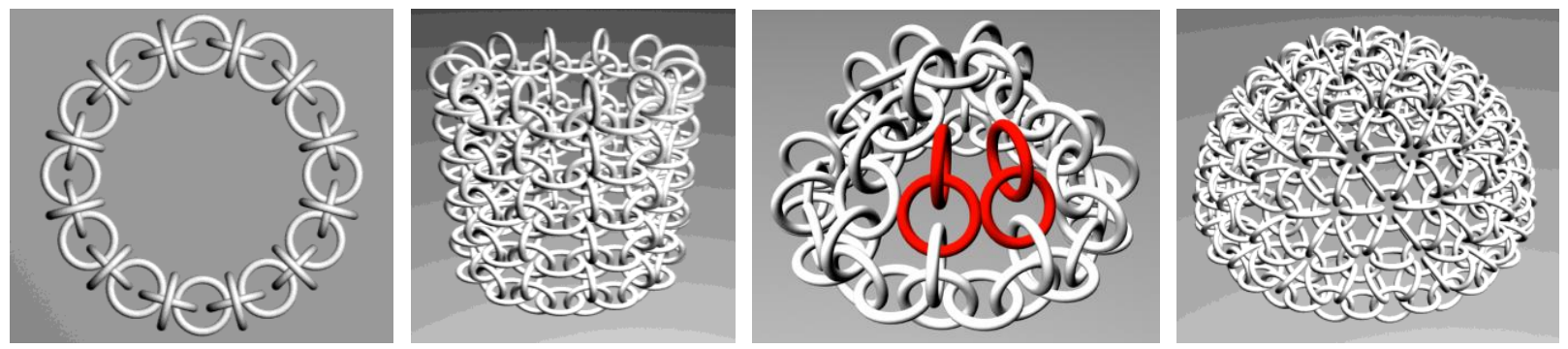

Figure 3: Cylindrical and hemispherical AM textile samples

Further investigation revealed the generation of a hemispherical AM textile sample could only be achieved through the use of a triangular-based system AM textile link configuration (instead of a quadrilateral-based) and the acceptance of dimensional variance within the individual link structures. The modelling of the hemispherical AM textile sample demonstrated in Figure 3 also required a significant amount of manual manipulation by the CAD user and was therefore laborious and error prone. While this can be tolerated for smaller AM textile samples, the modelling of larger AM textile structures, for example apparel, would be impractical.

In theory, an individual textile link structure can be designed with an extremely high level of complexity. However, this early expert modelling has demonstrated the difficulty encountered when 
attempting to combine these links into conformal or net-shape AM textile structures. In this case, the requirement is not the creation of a singular or linear and circular array of complex individual geometries, but the automated creation of a uniformly distributed collection of complex geometries, potentially numbering in the thousands, that ultimately create the hierarchy of a conformal AM textile. As complex (curved) geometries, for example a hemisphere, cannot be accurately described by any simple array or patterning function within current 3D CAD systems and no automated mapping function currently exists to replace the required manual manipulation of individual link geometries, the generation of conformal AM textile data is currently severely restricted. The work presented here has developed a novel approach to reduce these restrictions and quickly and efficiently model complex textile structures.

\subsection{AM textile modelling strategy}

In order to unlock the potential of conformal AM textiles and provide practical access to the efficient generation of AM textile data, a dedicated modelling strategy is required, with specific constraints:

- Provide an efficient means of generating complex conformal AM textile data suitable for manufacture by AM techniques

- Maintain uniformity within the dimensions of the individual AM textile link structures

- Remove the need for manual intervention by the user

Uniformity within the final AM textile structure is desirable due to manufacturing constraints and the potential relationship between geometry (size, shape) and functionality when considering highperformance AM textile structures [Bingham 2007]. Major dimensional variation within the individual AM textile links could result in either a failure to build (the links being too small, highly deformed or touching another link) or failure to perform as designed (scale, shape and functionality).

Observations from experimental modelling using current 3D CAD systems highlighted the efficiency of using an array or patterning function to describe the intended locations of a link structure and copying or repeating the original link geometry to those intended locations, as demonstrated in Figure 4, creating the final AM textile structure.
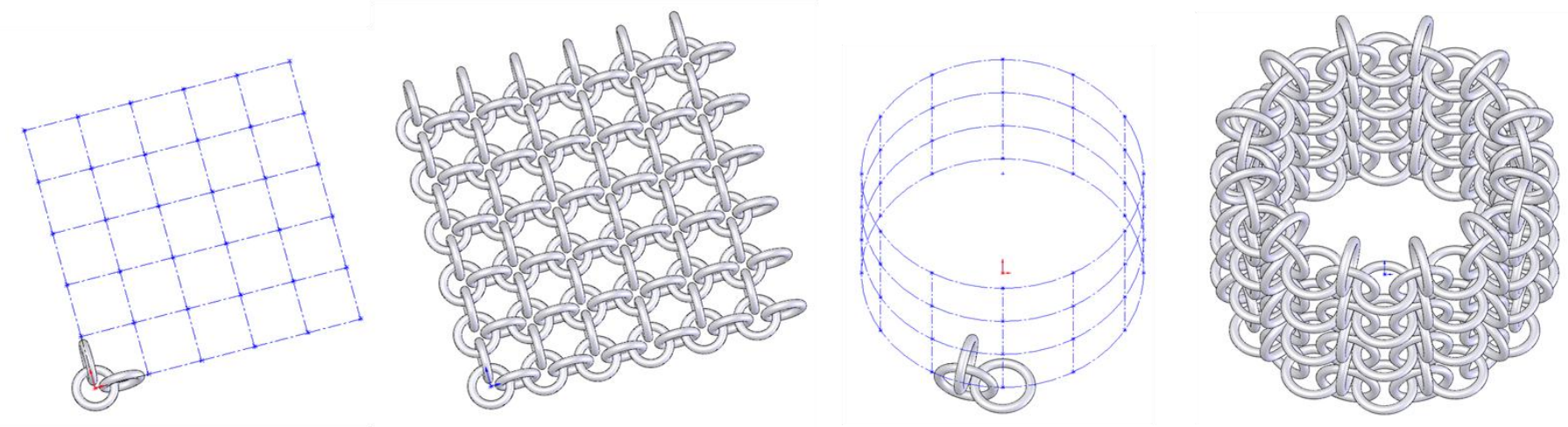

Figure 4: Array functioning (quadrilateral-based)

An efficient modelling strategy removes the requirement of any potential manual manipulation of the AM textile link structures by the CAD user and accurately controls the location and spacing between the individual geometries. Repeating the original link structure also removes any potential for dimensional variance within the resultant AM textile. The intended locations of the individual 
links (shown as dashed lines) in Figure 4 can be considered as a surface mesh representing the desired final configuration of the conformal or net-shape AM textile and developed the idea of generating and utilising a 'mapping' surface mesh for the generation of conformal and net-shaped AM textiles. However, while the strategy of generating a surface mapping mesh is relatively simplistic for planar and cylindrical samples as demonstrated in Figure 4, the generation of a mapping mesh for complex topology is much more complex. The first stage of development for the new modelling strategy was therefore the creation of a mapping mesh over complex topology.

\subsection{Mapping surface mesh}

The main requirement of the surface mapping mesh, when used as a means of providing locations over complex topology for the accurate positioning of AM textile link geometries, was for the mesh to have a uniform structure and equidistant node spacing as demonstrated in Figure 5.

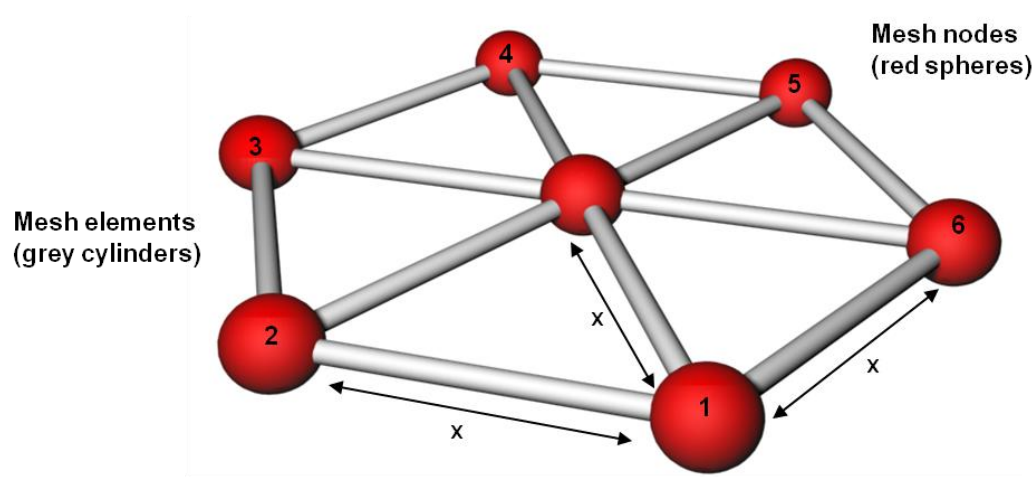

Figure 5: Idealised mapping mesh

For a surface mesh to exhibit such qualities, the only possible structure the mesh can be generated from, is a set of triangular elements. To clarify, if a section of a uniform and equidistant surface mesh is considered, as demonstrated in Figure 5 , the structure of the mesh can be described through a system of nodes and elements. The nodes of the mesh, shown as red spheres in Figure 5, are locations from the intended topology that are described by the mesh. The elements of the mesh, shown as grey cylindrical geometries in Figure 5, are described as the geometry connecting the nodes of the mesh, completing the surface mesh structure. For the surface mesh to be uniform, each node contained within the mesh must be connected to a maximum of six other nodes in a triangular, hexagonal configuration. Also, for the mesh to be equidistant, the distance observed between each node, shown as (x) in Figure 5 must all be constant, and therefore, the elements of the mesh must all be equal length.

The nodes and elements of the surface mesh therefore provide locations on the topology where AM textile link geometries could be positioned, as demonstrated in Figure 6. By mapping one link geometry configuration to the nodes of the surface mesh and a second link geometry configuration to the midpoints of the elements, an AM textile structure can be readily created (Figure 6). If a more complex AM textile configuration was utilised that only required one link structure, then this could also be achieved by additionally mapping to the node or element midpoints, as demonstrated in Figure 6. 

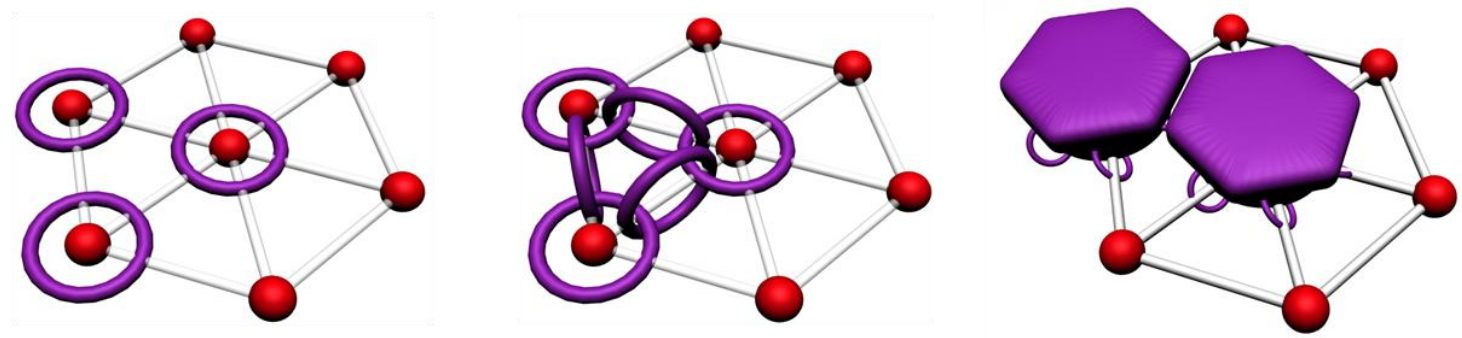

Figure 6: Mapping to a mesh

Various existing surface mesh generation algorithms were investigated for their application as a mapping mesh generator for AM textile data generation, including:

- Indirect or parametric space types [Marur 2005]

- Advancing front types [Borouchaki 2000]

- Direct and indirect sphere packing types [Shimada 1997]

Additionally, several commercially available Finite Element (FE) pre-processors were also explored, including:

- Fluent Gambit [Fluent 2011]

- MSC Patran [MSC 2011]

- ANSY [ANSY 2011]

- Hypermesh [Hypermesh 2011]

However, it was quickly established that the generation of a completely uniform and equidistant mesh structure as demonstrated in Figure 7 was not possible and a dedicated surface meshing algorithm was required.

Further research was undertaken to achieve this aim and a new dedicated surface meshing algorithm based on sphere packing [Shimada 1997] was developed by the author specifically for AM textile data generation [Bingham 2007]. The results of the research are demonstrated in Figure 7 for a range of topology and show the desired uniform and equidistant mesh structure required when utilised as a mapping mesh for AM textile data generation.
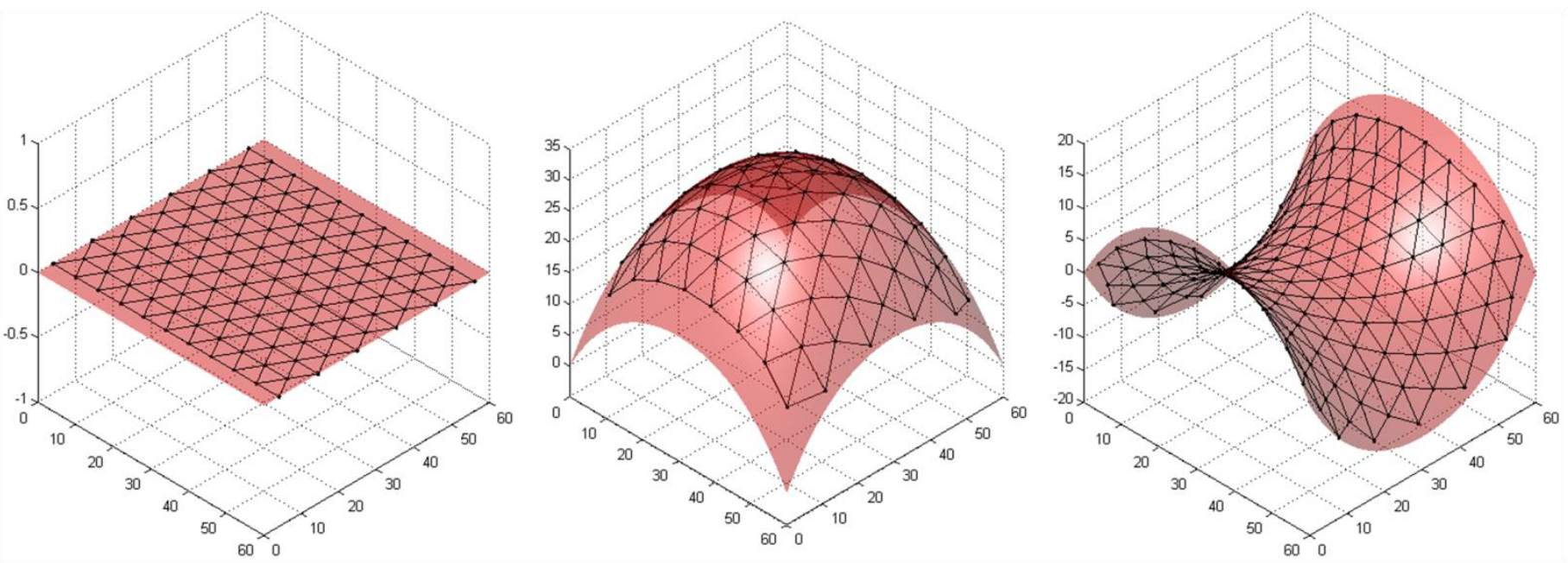

Figure 7: Example uniform and equidistant surface meshes 


\subsection{Development of a mapping tool for AM textile data generation}

The initial 3D modelling of AM textile structures highlighted many limitations when generating conformal or net-shaped AM textiles and the requirement for a dedicated 3D modelling strategy or tool. However, it also reinforced the geometric complexity achievable when considering the design and $3 \mathrm{D}$ modelling of individual AM textile link structures and the efficiency of mapping to a predefined mesh or grid. A requirement of any specifically developed modelling strategy or tool for the generation of AM textile data must also provide this level of geometric complexity for the design and development of present and future AM textiles. It was therefore decided to incorporate the capabilities of 3D CAD systems into the modelling strategy by simply importing the 3D data they generate. Similarly, it was decided not to directly incorporate the surface meshing algorithm discussed previously into the methodology but supply the necessary data as a further input. These decisions simplify the requirements of a dedicated tool for the generation of conformal AM textile data into a system capable of mapping complex 3D data to an imported surface mapping mesh. The stages of the mapping methodology can be summarised in the following four stages.

- Stage one: Import CAD data and surface mesh data

- Stage two: Calculate surface normals and mapping locations from surface mesh

- Stage three: Orientate link geometry to match mapping locations of the surface mesh

- Stage four: Translate orientated link to mapping location

The next sections of this paper relate to the four stages of the mapping methodology discussed, detailing and developing the mathematical principles and equations necessary for the successful mapping of one AM textile link structure. Creating a methodology for the successful mapping of one AM textile link structure to the node or element midpoint of a surface mesh can therefore be easily extended to produce a complete AM textile structure.

\subsection{Stage one: Import CAD data and surface mesh data}

The initial step of the mapping process required the import of $A M$ textile link 3D CAD data. As the ultimate aim of the mapping tool was the creation of a complete AM textile STL data file suitable for manufacture, it was appropriate to use the STL file format as the direct input for the mapping process. Using the STL format removed any necessity for data format conversion later in the process.

The STL file format represents the geometry it is describing by a system of vertices and triangles. Each triangle is described individually by three vertices which are described as Cartesian coordinates. An extract of a single triangle from the ASCII STL format is demonstrated in Figure 8.

\begin{tabular}{|c|c|c|c|c|}
\hline $\begin{array}{l}\text { Solid: Name } \\
\text { Facet normal } \\
\text { Outer loop }\end{array}$ & $\mathrm{n}(\mathrm{i})$ & $\mathrm{n}(\mathrm{j})$ & $n(k)$ & $\begin{array}{l}: \text { File name of geometry } \\
: \text { Unit normal components of triangle } \\
: \text { Start of triangle creation }\end{array}$ \\
\hline Vertex & $1(x)$ & $1(y)$ & $1(z)$ & : Vertex $1 \mathrm{x}, \mathrm{y}$ and $\mathrm{z}$ coordinates \\
\hline Vertex & $2(x)$ & 2(y) & $2(z)$ & : Vertex $2 x, y$ and $z$ coordinates \\
\hline Vertex & $3(x)$ & $3(y)$ & $3(z)$ & : Vertex $3 x, y$ and $z$ coordinates \\
\hline $\begin{array}{l}\text { End loop } \\
\text { End facet } \\
\text { End solid }\end{array}$ & & & & $\begin{array}{l}\text { : End triangle creation } \\
\text { : Triangle complete } \\
\text { : Geometry complete }\end{array}$ \\
\hline
\end{tabular}

Figure 8: Extract from ASCII STL data format of a single triangle 
Using the STL file format for CAD geometry import meant the only requirement of the mapping tool was the capability to read the coordinates of the vertices. Recreating each vertex sequentially, and therefore each triangle, will ultimately lead to the recreation of the entire AM textile STL link geometry.

The second requirement of the mapping tool was the capability to read the data contained within the mesh file. An important consideration here was the ability to accurately calculate the surface normal at every potential mapping location. Surface normal calculation was essential for ultimately generating a conformal AM textile structure that would both tessellate correctly and mimic the curvature of the intended surface topology of the final AM textile.

Generic FE mesh data files, in a similar manner to the STL file, consists of a node list with the corresponding Cartesian coordinates set to a global coordinate system, and a further list detailing which nodes are connected to each other. The second list of node connectivity therefore describes the element geometries of the surface mesh. Again, similar to the STL file usage, the mapping tool required only the capability to read the coordinates of the nodes and document which other nodes are connected to them within the surface mesh.

\subsection{Stage two: Calculate surface normals and mapping locations from surface mesh}

Calculating the surface normal and local coordinate system at the nodes and element midpoints required the vectors describing the elements connected to a particular node to be known. While this data was not included in the surface mesh file it could be easily calculated through the node connectivity list and the application of simple vector analysis. Figure 9 shows an example surface with a small section of a corresponding example surface mesh. The example mesh consists of nodes (A-G) and elements (ab-ag). The coordinates for the nodes (A-G) are supplied within the mesh data file with a further list detailing which nodes are connected to node (A).
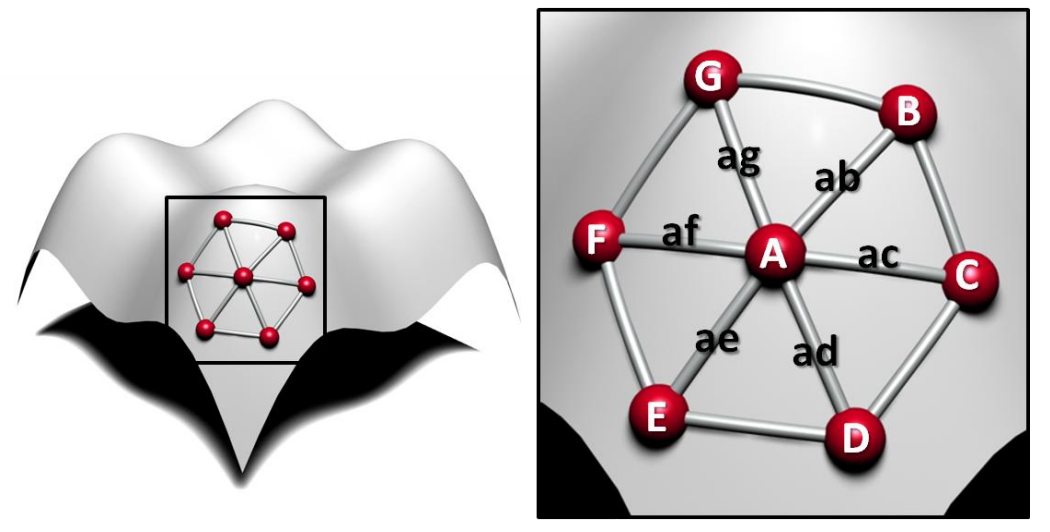

Figure 9: Surface mesh section

The vectors of the elements (ab-ag) can then be calculated using vector analysis and the node coordinates from the mesh file. Once the element vectors were calculated, it was then possible to calculate the surface normal at node (A) by calculating the vector product of pairs of connected vector elements. The vector product (sometimes described as the cross product) can be defined as a third vector perpendicular or normal to two input vectors. The notation for the cross product of two input vectors $\mathbf{a}$ and $\mathbf{b}$ for example, becomes $|\mathbf{a} \mathbf{x} \mathbf{b}|$, as shown in Figure 10. 


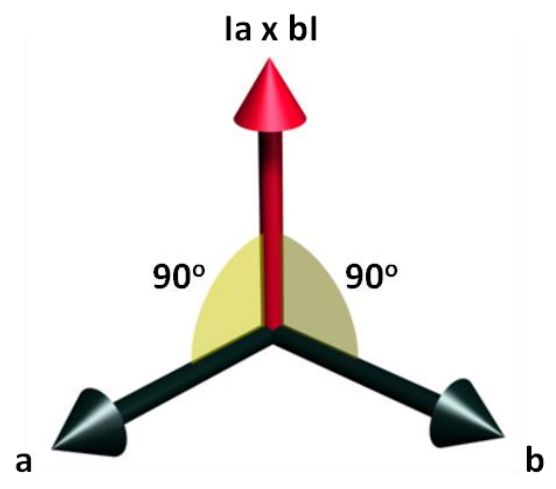

Figure 10: Vector cross product

$|\mathbf{a} \times \mathbf{b}|$ can be calculated using the determinant of the following matrix:

$$
|a \times b|=\left[\begin{array}{ccc}
i & j & k \\
x 1 & y 1 & z 1 \\
x 2 & y 2 & z 2
\end{array}\right]
$$

Where the input vectors $\mathbf{a}$ and $\mathbf{b}$ are described as:

$$
\begin{aligned}
& a=(x 1, y 1, z 1) \\
& b=(x 2, y 2, z 2)
\end{aligned}
$$

Therefore: $\quad|a \times b|=i\left[\begin{array}{ll}y 1 & z 1 \\ y 2 & z 2\end{array}\right]-j\left[\begin{array}{ll}x 1 & z 1 \\ x 2 & z 2\end{array}\right]+k\left[\begin{array}{ll}x 1 & y 1 \\ x 2 & y 2\end{array}\right]$

$$
|a \times b|=i(y 1 z 2-y 2 z 1)-j(x 1 z 2-x 2 z 1)+k(x 1 y 2-x 2 y 1)
$$

This equation must therefore be executed for every adjacent pair of non-parallel elements connected to node $(\mathbf{A})$ in the example surface mesh to produce a third vector perpendicular to both input vectors. Once completed, a series of six vectors are created from which an average can be taken that corresponds to the surface normal at the example node location (demonstrated in Figure $11)$.

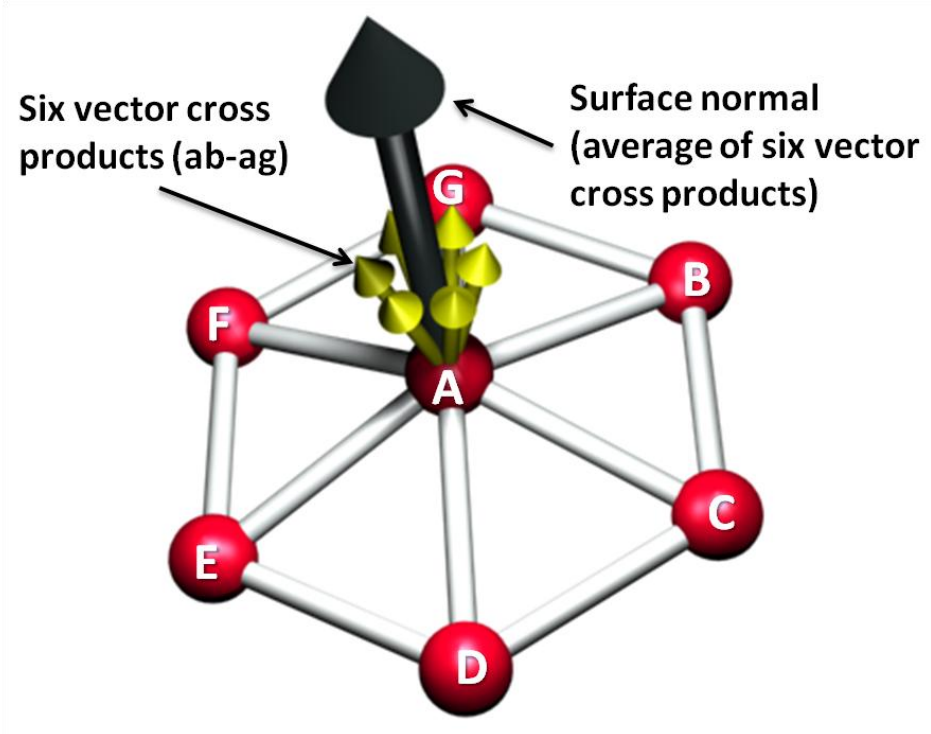

Figure 11: Calculation of surface normal 
The surface normal created at the example node location therefore became the $\mathbf{Z}^{\prime}$ component of the new local coordinate system at node (A) required to successfully orientate the link geometry from its original orientation. The $\mathbf{Y}^{\prime}$ component of the local coordinate system was calculated using the cross product of the surface normal $\left(\mathbf{Z}^{\prime}\right)$ and one existing mesh element vector that temporarily approximates both the global $\mathbf{X}$ axis and the $\mathbf{X}^{\prime}$ component of the local system (demonstrated in Figure 12). The accurate $\mathbf{X}^{\prime}$ component of the local system was finally calculated using the cross product of the $\mathbf{Z}^{\prime}$ component (surface normal) and the calculated $\mathbf{Y}^{\prime}$ component, also demonstrated in Figure 12.
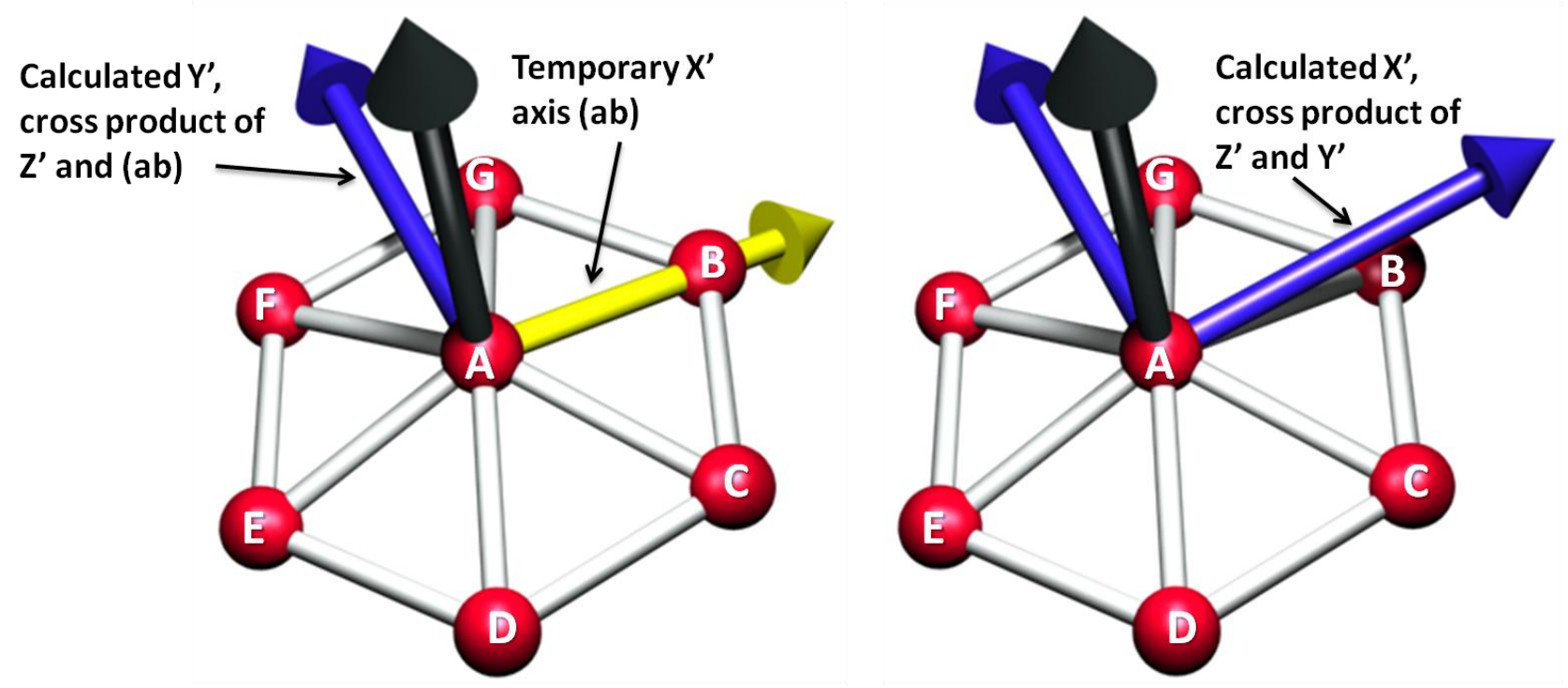

Figure 12: Calculation of local coordinate system

Using the equations and methodology developed it was possible to calculate a local coordinate system at every surface mesh node from generic mesh data for the purpose of mapping complex geometry. While not included within this example, the development of a local coordinate system for the midpoints of mesh elements, for example (ab) as demonstrated in Figure 10, can be achieved by first calculating the midpoint of the element using vector analysis and the local coordinate systems at both nodes (A) and (B), or any connected nodes that describe the element. Once achieved, the local coordinate system at the midpoint of the mesh elements can be determined by averaging the appropriate vectors from both the calculated local coordinate systems at both nodes which define the element.

\subsection{Stage three: Orientate link geometry to match mapping locations of the surface mesh}

Link orientation was achieved by transforming (rotating) the individual vertices contained within the STL file from their initial global coordinate system (described as $\mathbf{x}, \mathbf{y}, \mathbf{z}$ ) to the new local system at the mapping location (finally described as $\mathbf{x}^{\prime}, \mathbf{y}^{\prime}, \mathbf{z}^{\prime}$ ). Manipulation of the individual vertex coordinates, while retaining their connectivity information from the STL data, where each triangle is defined by its own section of code, enables the recreation of the individual triangles of the geometry in a new orientation. This was achieved by calculating the observed transformation (rotation) between the global coordinate system $(\mathbf{X}, \mathbf{Y}, \mathbf{Z})$ and the calculated local coordinate system $\left(\mathbf{X}^{\prime}, \mathbf{Y}^{\prime}, \mathbf{Z}^{\prime}\right)$ if it was located at the global origin, as demonstrated in Figure 13. 


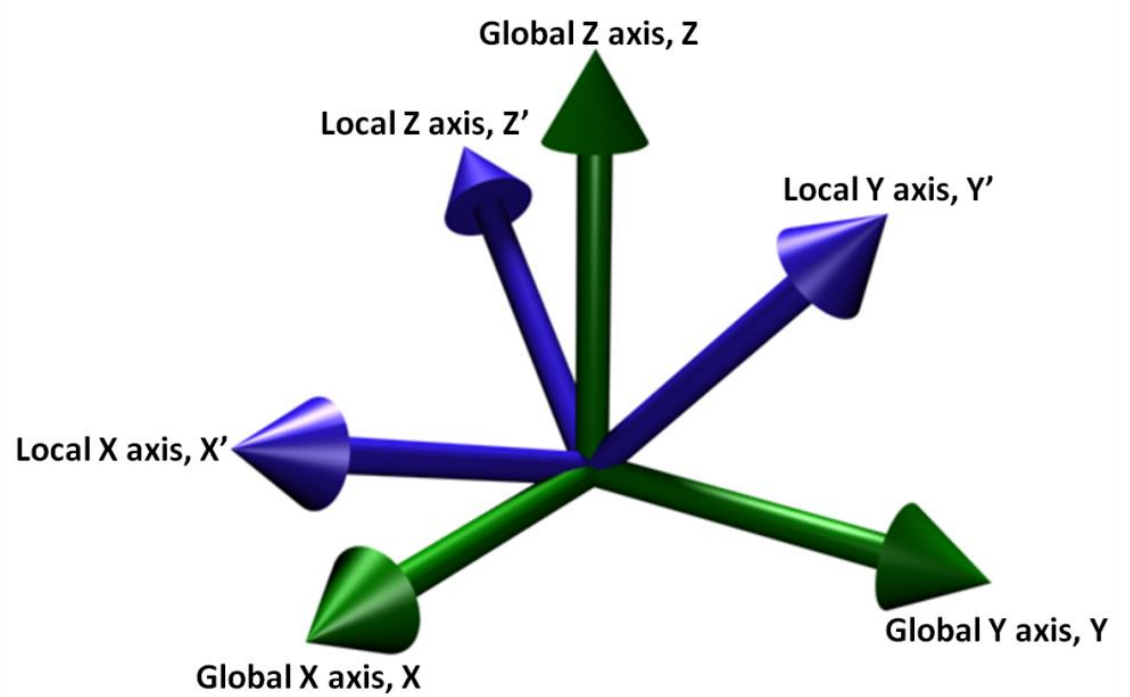

Figure 13: 3D transformation between coordinate systems

Using Vector analysis it was possible to generate a rotation matrix for the transformation between the two coordinate systems using the unit vectors components $(\mathbf{i}, \mathbf{j}, \mathbf{k})$ of the local coordinate system $\left(\widehat{\boldsymbol{X}}^{\prime}, \widehat{\boldsymbol{Y}}^{\prime} \& \widehat{\boldsymbol{Z}}^{\prime}\right)$. Matrix multiplication of the rotation matrix and the original vertex coordinates $(\mathbf{x}, \mathbf{y}, \mathbf{z})$ generated new orientated coordinates to match the local coordinate system as follows:

$$
\left[\begin{array}{l}
x^{\prime} \\
y^{\prime} \\
z^{\prime}
\end{array}\right]=\left[\begin{array}{lll}
\widehat{X}^{\prime}(\boldsymbol{i}) & \widehat{\boldsymbol{Y}}^{\prime}(\boldsymbol{i}) & \widehat{\boldsymbol{Z}}^{\prime}(\boldsymbol{i}) \\
\widehat{\boldsymbol{X}}^{\prime}(\boldsymbol{j}) & \widehat{\boldsymbol{Y}}^{\prime}(\boldsymbol{j}) & \widehat{\boldsymbol{Z}}^{\prime}(\boldsymbol{j}) \\
\widehat{\boldsymbol{X}}^{\prime}(\boldsymbol{k}) & \widehat{\boldsymbol{Y}}^{\prime}(\boldsymbol{k}) & \widehat{\boldsymbol{Z}}^{\prime}(\boldsymbol{k})
\end{array}\right] \times\left[\begin{array}{c}
\boldsymbol{x} \\
\boldsymbol{y} \\
\boldsymbol{z}
\end{array}\right]
$$

Where:

$$
\begin{array}{ll}
\boldsymbol{x}^{\prime}, \boldsymbol{y}^{\prime} \& \mathbf{z}^{\prime} & \text { New coordinate of a vertex } \\
\boldsymbol{x}, \boldsymbol{y} \& \boldsymbol{Z} & \text { Original coordinate of a vertex } \\
\widehat{\boldsymbol{X}}^{\prime}, \widehat{\boldsymbol{Y}}^{\prime} \& \widehat{\boldsymbol{Z}}^{\prime} & \text { Unit vectors of the local coordinate system } \\
\boldsymbol{i}, \boldsymbol{j} \& \boldsymbol{k} & \text { Components of the unit vectors }
\end{array}
$$

The matrix equation can be simplified further into algebraic notation and an individual equation generated for each axes of the local coordinate system:

$$
\begin{aligned}
& x^{\prime}=x \times \widehat{X}(i)+y \times \widehat{Y}(i)+z \times \widehat{Z}(i) \\
& y^{\prime}=x \times \widehat{X}(j)+y \times \widehat{Y}(j)+z \times \widehat{Z}(j) \\
& z^{\prime}=x \times \widehat{X}(k)+y \times \widehat{Y}(k)+z \times \widehat{Z}(k)
\end{aligned}
$$

These equations therefore provided a method for calculating the orientation of a single vertex in an STL file from its initial global coordinate system at the global origin to any potential local coordinate system calculated from the surface mesh also at the global origin. Repeating this process for every vertex within an STL file while retaining its triangle connectivity information ultimately leads to the recreation of the original STL geometry in a new orientation, while still remaining at the global origin. Having the capability to calculate the required local coordinate system at the surface mesh nodes and element midpoints, coupled with the equation to orientate the geometry to match such local systems, the last requirement of the mapping tool was the translation of the orientated geometry to the surface mesh node or element midpoints. 


\subsection{Stage four: Translate orientated link to mapping location}

The final stage required the translation of the newly orientated link geometry to the actual mapping location. The translation of the mapping geometry was achieved by translating the individual vertices of the STL file from their new orientation position at the global origin to the final mapping location as demonstrated in Figure 14. Once again this was achieved by translating the individual vertices of the STL while retaining the individual triangle connectivity. Repeating this process for every vertex within the STL file ultimately leads to the recreation of the entire geometry at the required mapping location.

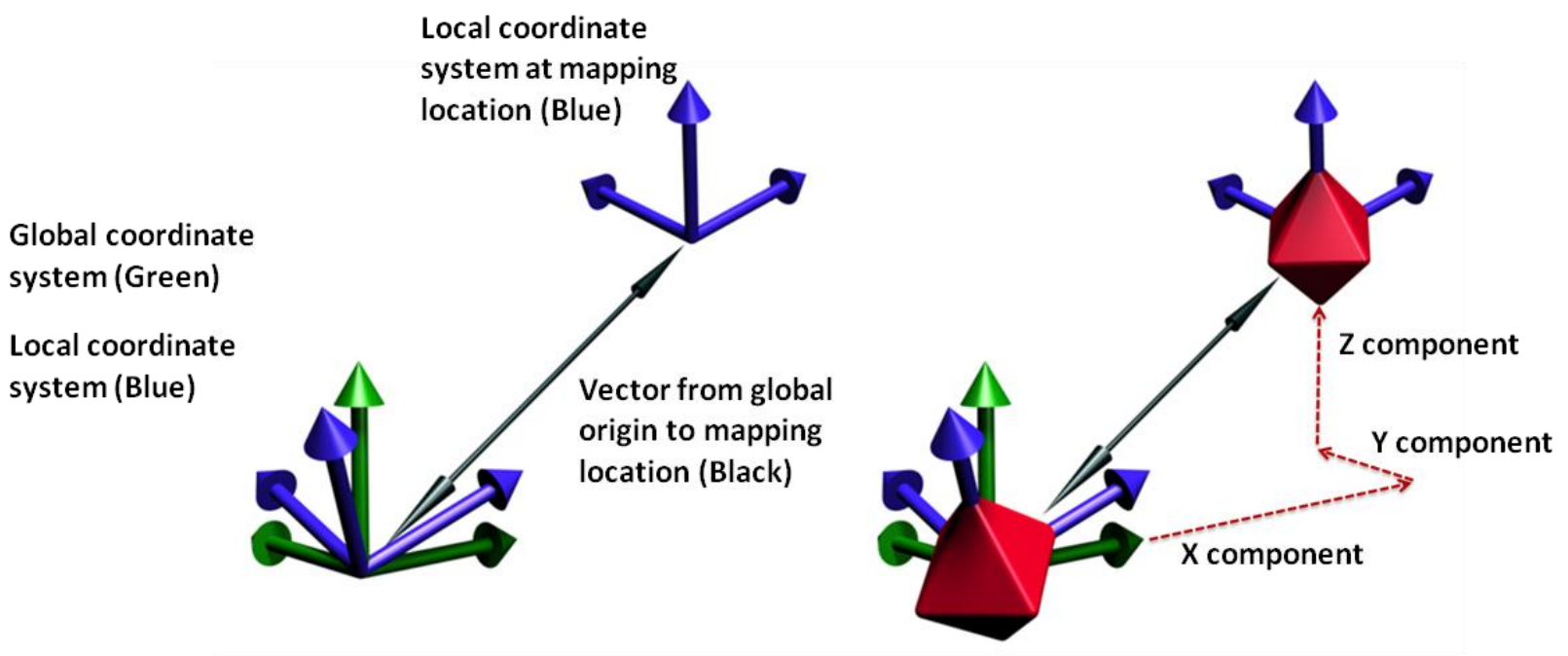

Figure 14: Vector translation

The translation of the geometry was achieved by using the vector from the global origin to the mapping location. As a 3D coordinate system was being utilised, the vector describing the mapping location included three components $(\mathbf{x}, \mathbf{y} \mathbf{\&} \mathbf{z})$ as demonstrate in Figure 14 . Therefore, by adding the relevant component of the vector to the relevant coordinate of the orientated vertex, the vertex can be translated to the correct position at the mapping location. This operation therefore concludes the process required by the mapping tool for the successful mapping of a single AM textile structure to a surface mesh. Repeating the methodology for every surface mesh node and or element midpoint contained within a targeted mapping mesh therefore enables the creation of a conformal AM textile structure.

The final requirement of the mapping tool was the creation of the STL file required for manufacture of the complete AM textile structure. As the connectivity of the original STL file describing a single AM textile link structure has been maintained throughout the developed mapping process, the mapping tool need only document the individually mapped copies as 'shell' entities within a new STL file assembly while omitting the original structure. A shell entity of an STL file can be defined as a non-connected body. Therefore, each structure becomes a separate entity which is fully described within the final STL file and suitable for manufacture. 


\subsection{Summary of methodology}

The four individual stages of the mapping methodology discussed in sections $3.1-3.4$ provide the core functionality of the developed AM textile modelling strategy. The entire AM textile modelling strategy can be summarised as follows:

1. Design individual AM textile link structure using conventional CAD software

2. Export individual $A M$ textile link structure as STL data

3. Identify target surface topology for the final AM textile structure

4. Generate uniform and equidistant mapping mesh of target surface topology

5. Identify mapping locations (node and or element midpoints) and calculate surface normal from mapping mesh

6. Copy and orientate original AM textile link structure to match required surface normal at the mapping location while remaining at the global origin

7. Translate orientated $\mathbf{A M}$ textile link structure to mapping location

8. Repeat stages six and seven until every mapping location has been utilised

9. Export final AM textile as STL data while omitting original AM textile link structure

Stages five, six and seven of the developed methodology are illustrated in Figure 15.

Stage Five: Calculate surface

normal at mapping location

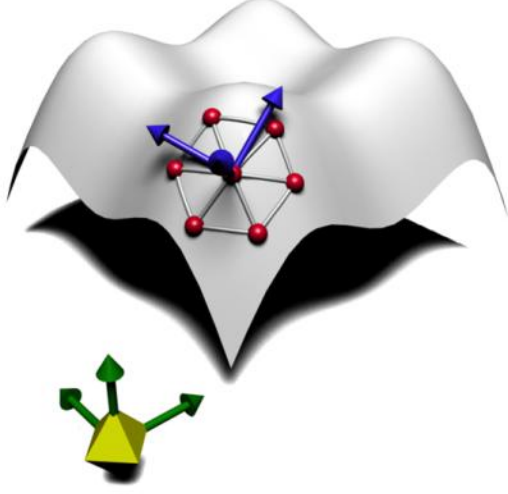

Stage Six: Orientate link structure at global origin

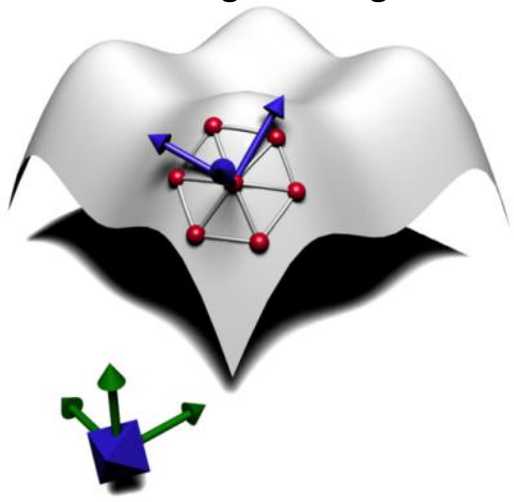

Stage Seven: Translate link structure to mapping location

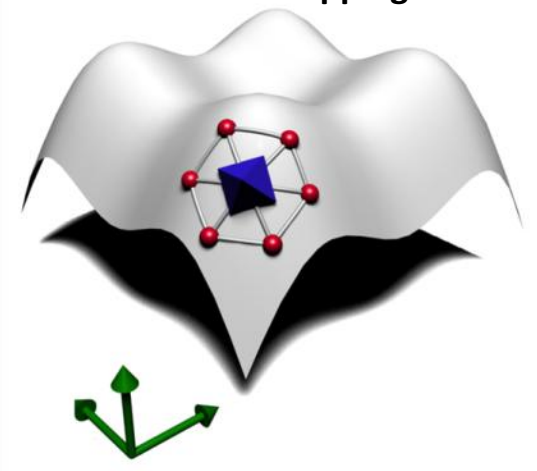

Figure 15: Left, stage five, Middle, stage six, Right, stage seven 


\subsection{Experimental testing of the mapping methodology}

To validate the capabilities of the documented methodology it was coded into a discrete software mapping tool. To expedite the coding process, the methodology developed was coded in collaboration with the University Nottingham who had the expertise and experience necessary to create such software [Nottingham 2011].

The software mapping tool has two key functions that required testing and validating:

- The ability to accurately calculate the surface normal at the mesh nodes and element midpoints

- The ability to accurately map complex geometry data described in STL format to the specified mapping locations without distortion

Successfully attaining both these capabilities would therefore provide all the functionality required to efficiently generate conformal AM textile data.

\subsection{Experiment one: Surface normal validation}

As previously discussed, the surface normal at any mesh node or element midpoint was calculated directly from the surface mesh and not the original geometry from which the mesh was created. To understand the effectiveness of the mapping tool in delivering this key capability, a marker geometry, as demonstrated in Figure 16, was mapped to example surface meshes demonstrated in Figure 16. The marker geometry was designed to allow a visual inspection of the final generated data to be undertaken as the direction of the mapped arrow indicated the surface normal at each mapping location. Any anomaly with the surface normal calculation at the mapping locations was then easily detected through the visual inspection of the arrow directions of the resultant STL data. The mapping meshes used for this experiment were:

- Planar mapping mesh: 60 units square with a 6 unit nodal spacing

- Domed (hemisphere approximation) mapping mesh: 60 units square with a 6 unit nodal spacing

- Saddle mapping mesh: 60 units square with a 6 unit nodal spacing
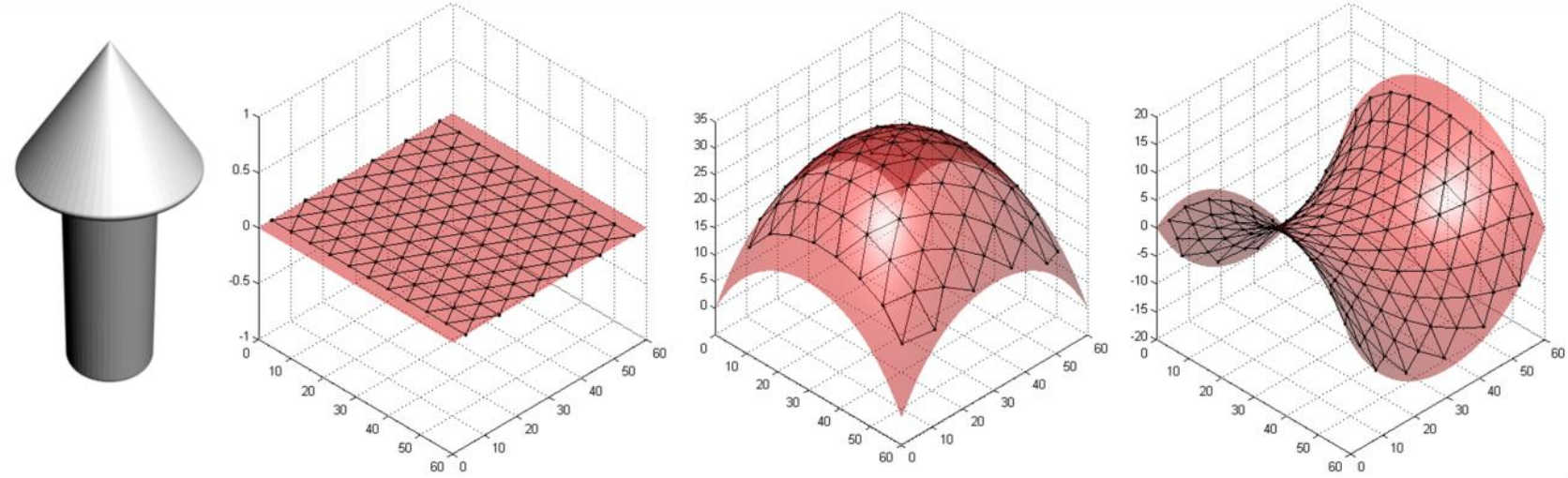

Figure 16: Marker geometry and three mapping meshes 


\subsubsection{Experiment one result}

The marker geometry demonstrated in Figure 16 was mapped to both the nodes and element midpoints of the three example surface meshes. The resultant STL data generated was then imported into 3D Studio Max [Autodesk 2011] to visual inspect the positions of the marker geometry relative to the mapping locations and the surface normal calculated at each location. The results of nodal mapping for each of the three surface meshes are demonstrated in Figure 17.

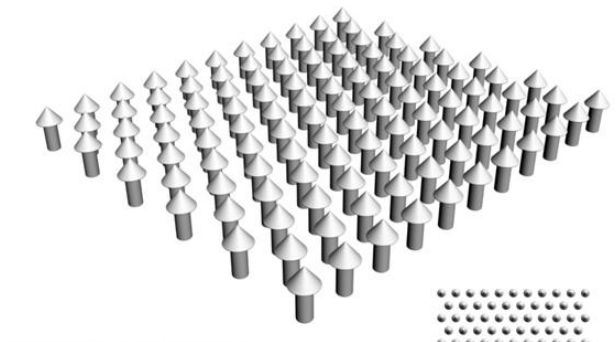

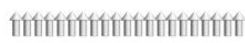

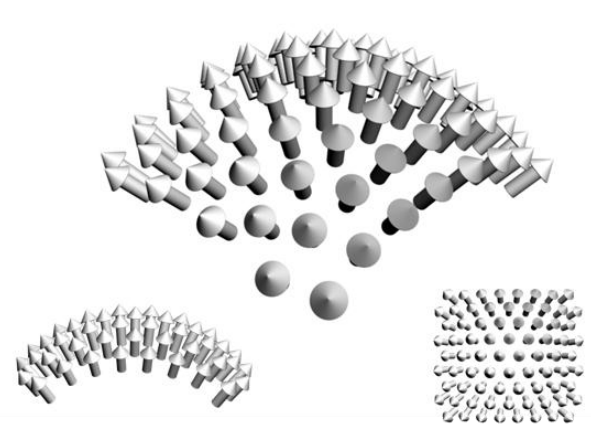

Figure 17: Marker geometry nodal mapping

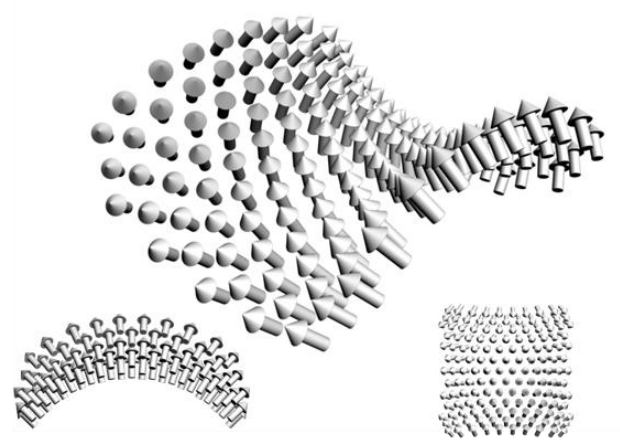

Figure 17 demonstrates that the orientation of the marker geometries accurately reflect the expected surface normal at the nodal mapping locations of the three meshes utilised. For each mapping mesh, the mapping tool accurately mapped the marker geometry to the intended mapping locations (nodes and element midpoints) while also matching the surface normal, resulting in a smooth transition of arrow direction and reflecting the curvature of the intended surface geometry.

\subsection{Experiment two: Complex geometry mapping}

The second experiment was conducted to determine whether the mapping methodology was capable of accurately mapping complex geometry without any distortion. Two complex test geometries where utilised for this experiment as demonstrated in Figure 18. Both test geometries, described as STL data, were mapped to each of the surface meshes detailed in experiment one (planar, dome and saddle), firstly to the nodes of the mapping mesh and then to the element midpoints.
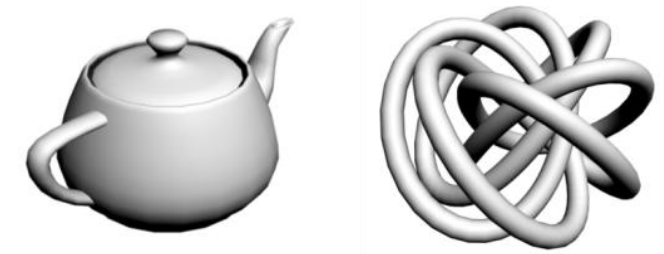

Figure 18: Left: Test geometry one, teapot. Right: test geometry two, mobius knot

\subsubsection{Experiment two result}

The results of the mapping experiment to the nodes of the example domed mapping mesh are demonstrated in Figure 19. For all three surface meshes utilised and both test geometries, the mapping methodology was successfully in accurately mapping the geometry to the nodes and element midpoints of the mesh by modifying the orientation to the required surface normal without 
distorting the original geometry. The individual shell entities within the generated STL data were extracted and compared with the original input STL data where no discernable differences were recorded.
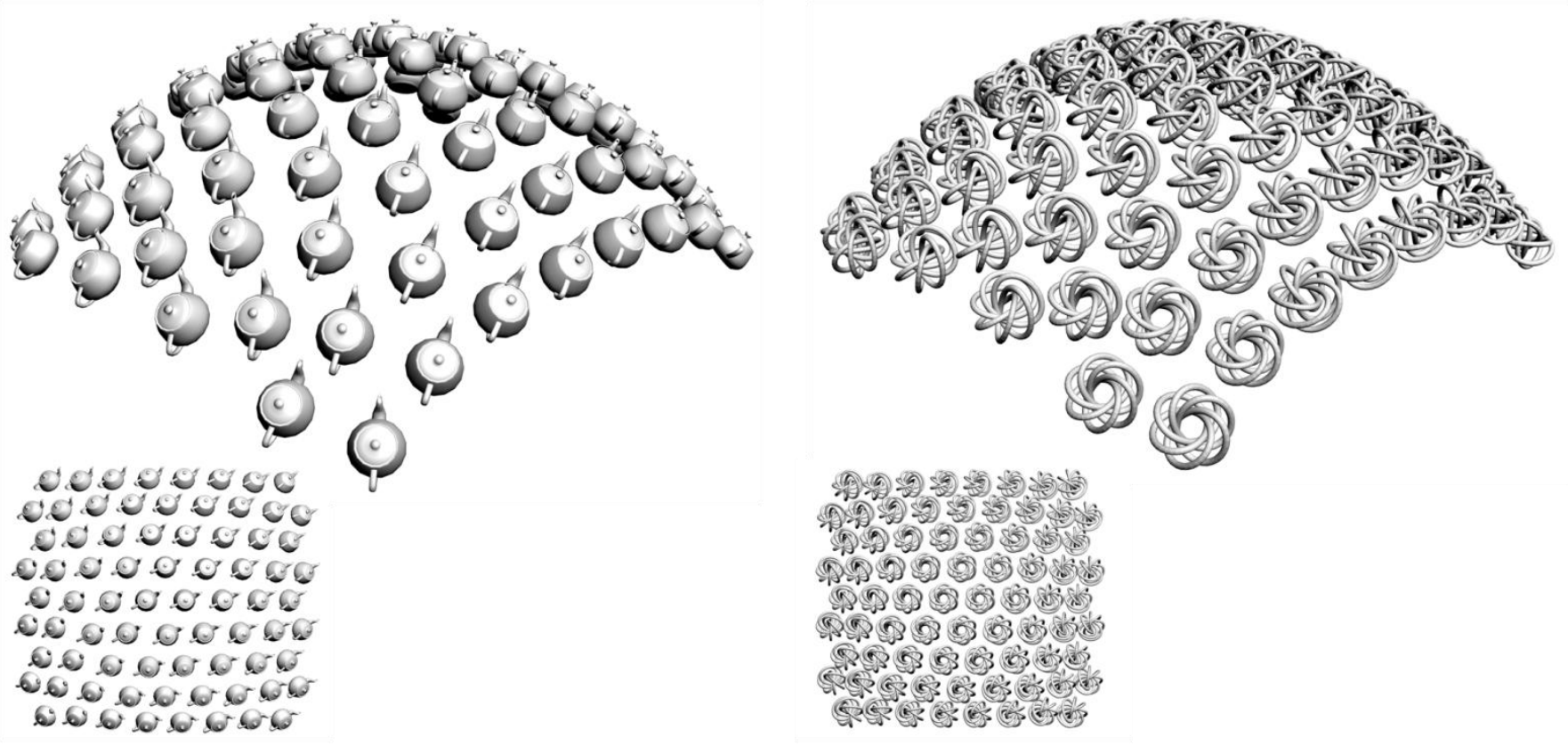

Figure 19: Experiment two nodal mapping to domed mesh

\subsection{Further validation}

The results of the two validation experiments indicate that the mapping methodology developed has the capability to accurately and efficiently map complex geometry described as STL data to a surface mesh without any distortion, whilst also matching orientation to the surface normal at each location. These capabilities therefore provide all the functionality required for the efficient generation of conformal AM textile structures. However, the accuracy of the generated data is dependent on the quality of the input mapping mesh and any distortion or irregularity within the mapping mesh is therefore replicated in the resultant data generated. Through the application of a uniform and equidistant mapping mesh it is possible to efficiently generate complex and conformal AM textile data suitable for manufacture. The results of this process are demonstrated in Figure 20, where a 1unit spacing mapping mesh based on a 60 unit square saddle surface has been utilised for the generation of a conformal AM textile sample. The modelling process for this sample required no user intervention other than the input of the original textile link structure (simple torus) as STL data and the surface mesh data file. The final STL data as demonstrated in Figure 20 was generated in under 30 seconds. 


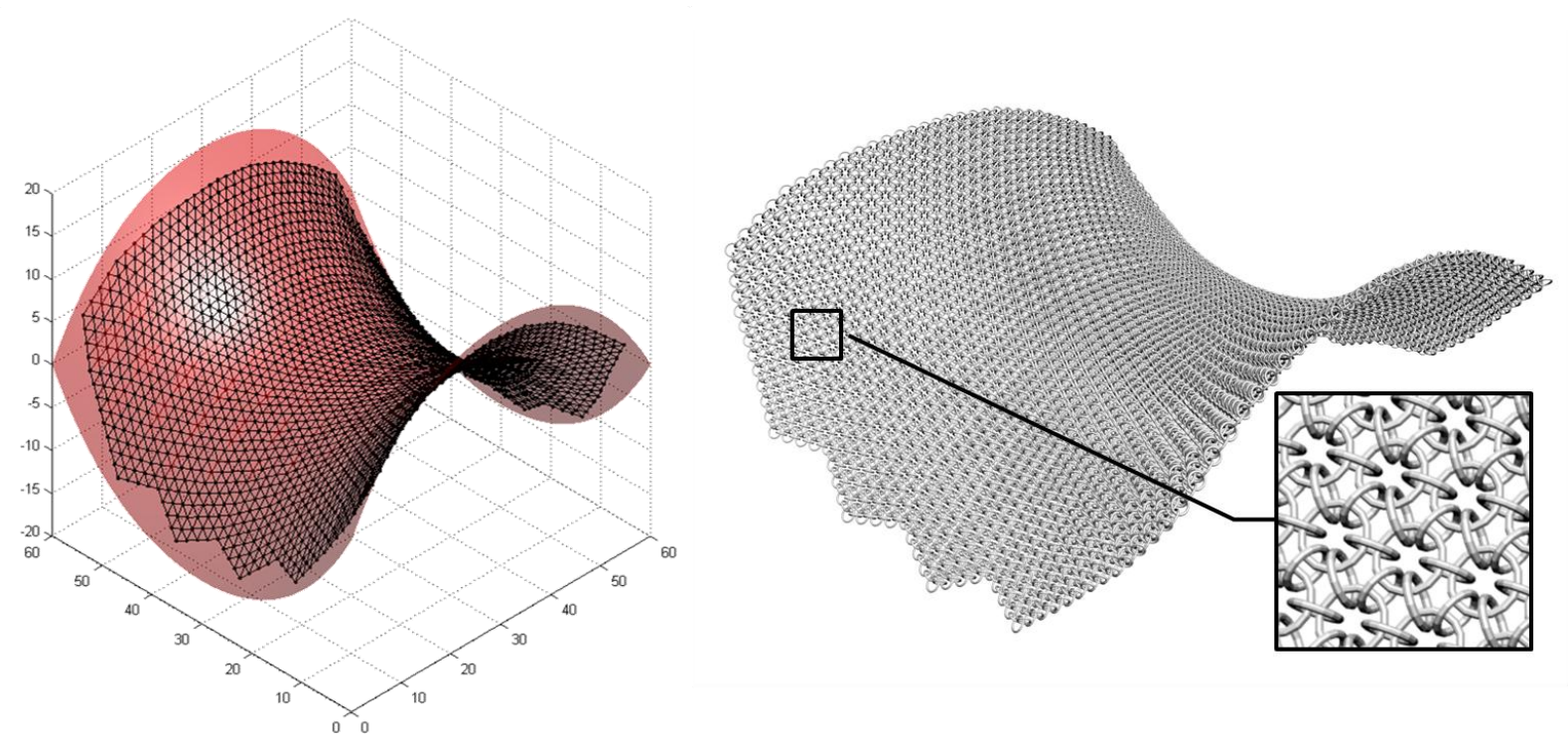

Figure 20: Conformal AM textile data generation using the developed mapping methodology

\subsection{Conclusion \& Further work}

The aim of this research was to investigate and develop an efficient means of generating conformal AM textile STL data suitable for manufacture. The research presented here demonstrates the issue surrounding the 3D modelling of conformal AM textiles using conventional 3D CAD systems and presents a robust methodology for the accurate and efficient generation of such complex 3D data.

The conclusions of the research can be summarised as follows:

- AM textiles provide a real opportunity for the design and manufacture of smart and highperformance textiles structures but practical access to an efficient means of generating their conformal and net-shape 3D data restricts wider scale adoption and investigation.

- Current CAD software solutions do not have the required capabilities to generate conformal AM textile data but do provide the modelling capabilities required for the design of complex individual link structures.

- Mapping complex geometries to a predefined surface mesh is a highly efficient means of accurately positioning large numbers of individual elements without user intervention.

- The presented methodology allows any complexity of geometry described as STL data to be accurately mapped to an input surface mesh without any user intervention.

- The presented methodology allows the generation of uniform and equidistant, conformal AM textile structures to be efficiently generated that are suitable for manufacture by AM techniques.

\subsection{Further work}

The presented methodology allows the efficient generation of conformal AM textile data. However, the suitability of the data generated is directly dependent on the quality of the input surface mesh. As discussed, any distortion or irregularity apparent within the input mesh will be replicated in the resultant AM textile data. While a certain level of irregularity of structure (uniformity) and nodal spacing variation (equidistance) can be tolerated for certain applications of AM textiles, this research was concerned with the generation of fully uniform and equidistant AM textile structures for functional applications where distortion of the link structures and nodal spacing variation was undesirable. While a new meshing algorithm has been specifically developed for this task, further 
work is required to extend its capabilities. The presented methodology is also compatible with a variety of alternative surface mesh formats to allow the generation of net shape AM textile samples beyond the capabilities of the specially developed meshing algorithm. Further work is also required to explore the design and application of AM textile possibilities that this new methodology enables. 


\subsection{References}

ANSYS software website, http://ansys.com/, accessed September 2006

Autodesk, 3D Studio Max software website, http://www.autodesk.co.uk/adsk/servlet/pc/index?sitelD=452932\&id=14596087, accessed August 2011

Bingham, G.A., Hague, R.J.M., Tuck, C.J., Long, A.C., Crookston, J.J. and Sherburn, M.N."Rapid Manufactured Textiles" International Journal of Computer Integrated Manufacture, Volume 20, Issue 1, 2007, pp96-105

Bingham, G.A., PhD thesis: “Generation of 3D data for Rapid Manufactured Textiles", July 2007, Loughborough University

Borouchaki, H., Laug, P. and George, P., "Parametric surface meshing using a combined advancingfront generalized Delaunay approach", International Journal of Numerical Methods in Engineering, v49, pp 233-259, 2000

Crookston, J.J., Long, A.C., Bingham, G.A. and Hague, R.J.M.,“Finite Element Modelling of Mechanical Behaviour of Rapid Manufactured Textiles". Proceedings of the Institution of Mechanical Engineers, Part L: Journal of Materials: Design and Applications, v 222, n 1, 2008, p 29-36

Fluent software website, http://www.ansys.com/Products/Simulation+Technology/Fluid+Dynamics/ANSYS+FLUENT, accessed July 2011

Hypermesh software website, http://www.altair.com/software/hw hm.htm , accessed August 2011 Johnson, A.J., Bingham, G.A. and Wimpenny, D., "Additive Manufactured Textiles for HighPerformance Stab resistant Applications" - 12th RDPM conference, Lancaster, England, June 2011 Marur, S., "On the meshing of trimmed 3D surfaces", Advances in Engineering Software, v36, pp 338-345, 2005)

MSC software website, http://www.mscsoftware.com/Products/CAE-Tools/Patran.aspx, accessed July 2011

Shimada, K., Yamada, A. and Itoh, T., "Anisotropic triangular meshing of parametric surfaces via close packing of ellipsoidal bubbles", Proceedings of the 6th International Meshing Roundtable, Park City, pp 375-390, 1997

Nottingham, Textile Composites Research Group at the University of Nottingham website, http://www.textiles.nottingham.ac.uk/, accessed August 2011 